Pacific Journal of Mathematics

A CONSTRUCTION OF AN ORDERED DIVISION RING WITH 


\title{
A CONSTRUCTION OF AN ORDERED DIVISION RING WITH A RANK ONE VALUATION
}

\author{
Ka Hin Leung
}

\begin{abstract}
Let $\left(k, P_{k}\right)$ be an ordered field and $\Gamma$ be a dense additive subgroup of $\mathbb{R}$. In this paper, we shall construct a noncommutative ordered division ring $(D, P)$ and a compatible valuation $v$ on $(D, P)$ such that (i) the value group of $v$ is $\Gamma$ and (ii) the residue division ring $\left(\bar{D}_{v}, \bar{P}_{v}\right)$ is order isomorphic to $\left(k, P_{k}\right)$. This problem is interesting because, in effect, we are constructing the "simplest" or in some sense the smallest noncommutative ordered division ring.
\end{abstract}

1. Introduction. Before we formulate the problem concerned in this paper, we first establish some basic terminologies.

Let $D$ be a division ring. A subset $P$ is called an ordering on $D$ if (i) $P+P \subset P$, (ii) $P \cdot P \subset P$, (iii) $P \cup(-P)=D$, (iv) $P \cap(-P)=\{0\}$. In this case we say $(D, P)$ is an ordered division ring, and write $a>_{P} b$ if $a-b \in P \backslash\{0\}$. (For convenience, we shall simply write $a>b$ if there is no confusion of the ordering concerned.) A valuation on $D$ is a surjective mapping $v: D \rightarrow G \cup\{\infty\}$, where $G$ is a totally ordered group (written additively though not necessarily abelian), such that for all $a, b \in D$,

(i) $v(a)=\infty$ if and only if $a=0$,

(ii) $v(a b)=v(a)+v(b)$,

(iii) $v(a+b) \geq \min \{v(a), v(b)\}$.

Also, we let $R_{v}:=\{a \in D: V(a) \geq 0\}$, the valuation ring of $v$; $I_{v}:=\{a \in D: v(a)>0\}$, the unique maximal left ideal and maximal right ideal of $R_{v} ; \bar{D}_{v}:=R_{v} / I_{v}$, the residue division ring of $v$ and $\pi_{v}: R_{v} \rightarrow \bar{D}_{v}$, the natural projection from $R_{v}$ to $\bar{D}_{v}$. For a reference, see [S: Chapter 1].

Next, we define the notion of compatibility of orderings and valuations on a division ring.

Definition 1.1. Let $v: D \rightarrow G \cup\{\infty\}$ be a valuation and $P$ an ordering on $D$. We say $v$ is compatible with the ordering $P$ if for any $a, b \in P \backslash\{0\}, a-b \in P \backslash\{0\}$ implies $v(a) \leq v(b)$ in $G$.

LeMMA 1.2 [T1: Lemma 3.4]. Let $(D, P)$ be an ordered division ring. Then $v$ is compatible with $P$ if and only if $1+I_{v} \subset P$. 
As a consequence of the above lemma, a valuation $v$ compatible with an ordering $P$ on a division ring $D$ induces an ordering

$$
\bar{P}_{v}:=\left\{a+I_{v}: a \in R_{v} \cap P\right\} \quad \text { on } \bar{D}_{v} \text { [T2: Section 0]. }
$$

There is also a natural compatible valuation $v_{P}$ associated with $P$. In fact, $v_{P}$ is the valuation induced by the valuation ring

$$
R_{v_{P}}:=\{a \in D: \exists n \in \mathbb{N} \text { with } n \pm a \in P\} .
$$

We call $v_{P}$ the natural valuation of $(D, P)$. For details, see [T1: Chapter 1, Theorem 3.5].

We are now ready to formulate the problem.

Problem 1.3. Given an ordered division ring $\left(k, P_{k}\right)$ and an ordered group $\Gamma$ do there exist a noncommutative ordered division ring $(D, P)$ and a compatible valuation $v$ on $(D, P)$ such that

(I) the value group of $v$ is $\Gamma$ and

(II) the residue division ring $\left(\bar{D}_{v}, \bar{P}_{v}\right)$ is order isomorphic to $\left(k, P_{k}\right)$ ?

The cases when $k$ is noncommutative or $\Gamma$ is nonabelian are simple. We can simply apply Neumann's construction ([N], [Sch: Theorem 1.10]) to get the desired $(D, P), v$. However, in case when $k$ is a field and $\Gamma$ is abelian, usually it is not easy to define suitable order automorphisms needed in Neumann's construction. In fact, the only order automorphism of any field $k \subset \mathbb{R}$ is the identity mapping. Furthermore, it can also be proved that such $(D, P), v$ do not exist in case $k$ is algebraic over $\mathbb{Q}$ and $\Gamma \cong \mathbb{Z}$. For detail, see [Sch: Chapter 3].

Problem 1.3 was first considered in M. Schröder's Münster paper [Sch]. In his paper he assumes $\left(k, P_{k}\right)$ is archimedean, and is able to construct $(D, P), v$ satisfying the above criteria in the following cases:

(A) the transcendence degree of $k$ over $\mathbb{Q}$ is at least 1 and $\Gamma$ is arbitrary.

(B) $k$ is algebraic over $\mathbb{Q}$ and $\Gamma$ is a dense subgroup of $(\mathbb{R},+)$ not contained in $\mathbb{Q}$.

For their proofs, see [Sch: Theorem 6.2, 6.6]. Note also that in these cases, the valuations involved are actually the natural valuations of $(D, P)$. 
It is not difficult to see that Schröder's method can be generalized to any ordered field $\left(k, P_{k}\right)$ in case $\Gamma$ is dense in $\mathbb{R}$ but not contained in $\mathbb{Q}$. Thus, we reduce Problem 1.3 to the case when $\Gamma$ is a dense subgroup of $\mathbb{Q}$. From now on, we shall fix an ordered field $\left(k, P_{k}\right)$ (not necessarily archimedean) and a dense subgroup $\Gamma$ in $(\mathbb{R},+)$. It is clear that we may always assume $\Gamma$ contains $\mathbb{Z}$. Our objective is to construct a noncommutative ordered division ring $(D, P)$ and a compatible valuation $v$ such that (I) and (II) are satisfied. Our strategy is as follows. Firstly, we shall construct a suitable ordered field $\left(K, P^{\prime}\right)$ and a compatible valuation $\phi$ on $K$, such that the conditions (I), (II) are satisfied. Then, we construct a suitable order automorphism $\sigma$ on $K$ and form the skew polynomial ring $K[t, \sigma]$. Since $K[t, \sigma]$ is an Ore domain, $D$, the ring of quotients, exists. $D$ can also be regarded as a division subring of $K((t, \sigma))$. It is well known that with respect to any ordering $Q$ on $K((t, \sigma)), t$ is infinitesimally small when compared with any positive element in $K$. Thus with respect to the ordering induced by $Q$ on $D$, the value group of the extension of $\phi$ will no longer be $\Gamma$. Naturally, we may ask if this is the only way to order $D$. Is it possible to define an ordering $P$ containing $P^{\prime}$ on $D$ such that $\phi$ extends to a compatible valuation $v$ on $(D, P)$ with its value group remaining unchanged? Our goal is to show that under some situations, the answer is affirmative. In those cases, $(D, P), v$ are what we want.

2. Fields of formal power series. From now on, we shall fix the following notation: $\left(k, P_{k}\right)$ is an ordered field; $\Gamma$ is a dense subgroup in $(\mathbb{R},+)$ containing $\mathbb{Z}$. In this section, our goal is to construct suitable $\left(K, P^{\prime}\right), \phi$ and $\sigma$ as defined above.

Let $F$ be a field and $G$ be an ordered abelian group. We denote the field of formal power series of $F$ over $G$ by $F((G))$, i.e.

$$
\begin{aligned}
F((G)):=\left\{\sum_{g \in G} a_{g} x^{g}:\right. & a_{g} \in F, g \in G \\
& \text { and } \left.\operatorname{supp}\left(\sum_{g \in G} a_{g} x^{g}\right) \text { is well ordered }\right\} .
\end{aligned}
$$

Here $\operatorname{supp}\left(\sum_{g \in G} a_{g} x^{g}\right):=\left\{g \in G: a_{g} \neq 0\right\}$. In $F((G))$, multiplication is induced by the rules that

$$
\forall g, h \in G, \quad a \in F, \quad x^{g} x^{h}=x^{g+h} \quad \text { and } \quad a x^{g}=x^{g} a .
$$


Let $\phi: F((G)) \rightarrow G \cup\{\infty\}$ be the mapping sending any $f \in F((G))^{*}$ to $\min \operatorname{supp}(f)$ and 0 to $\infty$. Then we have

Proposition 2.1. (i) $\phi$ is a valuation on $F((G))$ with value group $G$ and $\left.\pi_{\phi}\right|_{F}: F \rightarrow \overline{F((G))_{\phi}}$ is an isomorphism.

(ii) $F((G))$ is complete with respect to the value topology $T_{\phi}$.

(iii) Suppose $F$ is ordered. Then $P_{G}:=\left\{\alpha \in F((G)): a_{\phi(\alpha)}>0\right.$ in $F\} \cup\{0\}$ is an ordering compatible with $\phi$.

(iv) For any nonzero $x, y \in F((G))$, if $\phi(x-y)>\phi(y)$, then $\phi(x)=\phi(y)$ and $x y^{-1} \in P_{G}$ (i.e. $x, y$ are of the same sign with respect to the ordering $\left.P_{G}\right)$.

Proof. For (i) and (ii), see [P: Chapter II, §5, Theorem 8]. For (iii), see [P: Chapter II, $\S 5$, Theorem 6]. Note that our definition of $F((G))$ is slightly different from that in $[\mathbf{P}]$. In $[\mathbf{P}]$, the author uses anti-well ordered subsets (i.e. every subset contains a maximal element) instead. Of course, the argument still works in our case. Observe also that $x y^{-1}=1+(x-y) y^{-1}$ and by assumption $\phi\left((x-y) y^{-1}\right)>0$; therefore $\phi\left(x y^{-1}\right)=0$ and $(x-y) y^{-1} \in I_{\phi}$. It follows that $\phi(x)=\phi(y)$, and $x y^{-1}=1+(x-y) y^{-1} \in P_{G}$ by Lemma 1.2.

Let $F[G]:=\left\{\sum_{i=1}^{n} a_{i} x^{g_{i}}: a_{i} \in F, g_{i} \in G, n \in \mathbb{N}\right\}$ be the group ring of $G$ over $F$ and $F(G)$ be the quotient ring of $F[G]$ in $F((G))$. Let $F\langle G\rangle$ be the $T_{\phi}$ closure of $F(G)$ in $F((G))$. Before we state the next proposition, let us recall the definitions of completion and imbedding. For any valued field $(L, v)$, we call $(\widehat{L}, \hat{v})$ a completion of $(L, v)$ if $\widehat{L}$ is $T_{\hat{v}}$ complete; $L$ is $T_{\hat{v}}$ dense in $\widehat{L}$ and $\left.\hat{v}\right|_{L}=v$. Also, we call $\beta:\left(L^{\prime}, v^{\prime}\right) \rightarrow(L, v)$ an imbedding if $v \circ \beta=v^{\prime}$.

Proposition 2.2. Let $F, G, \phi$ be as before. Suppose $G$ is of rank one (i.e. $G$ can be order-embedded in $(\mathbb{R},+)$ ). Then

(i) $\left(F\langle G\rangle,\left.\phi\right|_{F\langle G\rangle}\right)$ is a completion of $\left(F(G),\left.\phi\right|_{F(G)}\right)$ and is therefore Henselian.

(ii) $F\langle G\rangle=\left\{\sum_{i=0}^{\infty} a_{i} x^{g_{\imath}}: a_{i} \in F, g_{i} \in G,\left(g_{i}\right)_{i \in \mathbb{N}}\right.$ is strictly increasing and divergent $\}$.

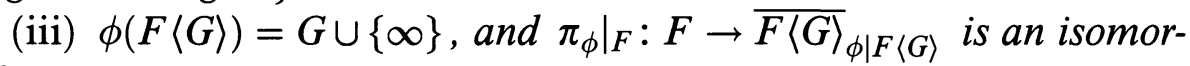
phism.

(iv) Suppose $F$ is ordered. Then $\left.\phi\right|_{F\langle G\rangle}$ is compatible with $P_{G} \cap$ $F\langle G\rangle$, where $P_{G}$ is as defined in Proposition 2.1(iii). 
Proof. (i) is a consequence of [E: Corollary 2.6] and [E: Theorem 17.18]. By (i), we see easily that the R.H.S. of (ii) is a subset of $F\langle G\rangle$. Therefore to show (ii), it suffices to show that for any $y \in F\langle G\rangle$, $g \in G,\{a \in \operatorname{supp} f: a<g\}$ is finite. This clearly is equivalent to the density of $F[G]$ in $F(G)$ and $F\langle G\rangle$.

It is clear that we only need to show that if $w=\sum_{i=1}^{m} a_{i} x^{g_{i}} \in F[G]$ with $g_{1}<g_{2}<\cdots<g_{m}$ in $G$ and $a_{1} \neq 0$, then $1 / w$ is a limit of a convergent sequence in $F[G]$. Let us write $w=a_{1} x^{g_{1}}\left(1+w^{\prime}\right)$ where $w^{\prime}=\sum_{i=2}^{m} a_{1}^{-1} a_{i} x^{g_{i}-g_{1}}$. For all $n \in \mathbb{N}$, we define

$$
w_{n}=a_{1}^{-1} x^{-g_{1}} \sum_{i=0}^{n}\left(-w^{\prime}\right)^{i} \in F[G] .
$$

As $\phi\left(w^{\prime}\right)>0$, it is obvious that the sequence $\left\{w_{n}\right\}_{n \in \mathbb{N}}$ converges to $1 / w$.

Lastly, (iii) is obvious and (iv) follows from Proposition 2.1(iii).

Lemma 2.3. Let $a>0$ be fixed in $G$ and $\gamma: F[G] \rightarrow F\langle G\rangle$ be $a$ ring homomorphism such that

$$
\forall y \in F[G] \backslash\{0\}, \quad \phi(\gamma(y)-y) \geq \phi(y)+a .
$$

Then $\gamma$ extends to a unique automorphism $\gamma^{\prime \prime}: F\langle G\rangle \rightarrow F\langle G\rangle$ such that

$$
\forall y \in F\langle G\rangle^{*}, \quad \phi\left(\gamma^{\prime \prime}(y)-y\right) \geq \phi(y)+a .
$$

Proof. Let $\gamma^{\prime}:\left(F(G),\left.\phi\right|_{F[G]}\right) \rightarrow\left(F\langle G\rangle,\left.\phi\right|_{F\langle G\rangle}\right)$ be the unique extension of $\gamma$. Notice that $\gamma^{\prime}$ is well defined as, by $(*), \gamma$ is injective. Also, Proposition 2.1 (iv) and (*) imply that $\phi \circ \gamma(x)=\phi(x)$ for all $x \in F[G]$. This implies $\phi \circ \gamma(x)=\phi(x)$ for all $x$ in $F(G)$. Next, we prove that $\gamma^{\prime}(F[G])$ is dense in $F\langle G\rangle$. It suffices to show that for any $y \in F\langle G\rangle^{*}, n \in \mathbb{N}$, there exists $y_{n} \in F[G]$ such that $\phi\left(y-\gamma\left(y_{n}\right)\right) \geq \phi(y)+n a$. We prove this by induction on $n$.

The density of $F[G]$ in $F\langle G\rangle$ implies the existence of $y_{1} \in F[G]$ such that $\phi\left(y-y_{1}\right) \geq \phi(y)+a$. Using $y-\gamma\left(y_{1}\right)=\left(y-y_{1}\right)+\left(y_{1}-\gamma\left(y_{1}\right)\right)$ and $(*)$, we get

$$
\phi\left(y-\gamma\left(y_{1}\right)\right) \geq \min \left\{\phi\left(y-y_{1}\right), \phi\left(\gamma\left(y_{1}\right)-y_{1}\right)\right\} \geq \phi(y)+a .
$$

Now suppose there exists $y_{n} \in F[G]$ such that $\phi\left(y-\gamma\left(y_{n}\right)\right) \geq \phi(y)+$ $n a$. After replacing $y$ by $y-\gamma\left(y_{n}\right)$ in the above argument, we obtain $w \in F[G]$ such that

$$
\phi\left(\left(y-\gamma\left(y_{n}\right)\right)-\gamma(w)\right) \geq \phi\left(y-\gamma\left(y_{n}\right)\right)+a \geq \phi(y)+(n+1) a .
$$

So we can simply take $y_{n+1}$ to be $y_{n}+w$. 
It follows that $\gamma^{\prime}(F[G])$ is dense in $F\langle G\rangle$. Hence, by [E: Corollary 2.4], $\gamma^{\prime}$ extends to a continuous automorphism $\gamma^{\prime \prime}$. It remains to prove that for any $y$ in $F\langle G\rangle, \phi\left(\gamma^{\prime \prime}(y)-y\right) \geq \phi(y)+a$. Recall that $F[G]$ is dense in $F\langle G\rangle$ and $\phi$ (see [E: (1.3)]), $\gamma^{\prime \prime}-1$ are continuous with respect to the value topology $T_{\phi \mid F\langle G\rangle}$. So there exists $w \in F[G]$ such that $\phi(w)=\phi(y)$ and $\phi\left(\left(\gamma^{\prime \prime}-1\right)(y-w)\right) \geq \phi(y)+a$. Therefore combining with the fact that $\phi(\gamma(w)-w) \geq \phi(w)+a$, we get

$$
\begin{aligned}
\phi\left(\gamma^{\prime \prime}(y)-y\right) & \geq \min \left\{\phi\left(\gamma^{\prime \prime}(y)-y+\gamma(w)-w\right), \phi(\gamma(w)-w)\right\} \\
& \geq \phi(y)+a .
\end{aligned}
$$

Let $\bar{k}$ be an algebraic closure of $k$ and $\Gamma_{c}$ be the divisible hull of $\Gamma$ in $\mathbb{R}$. We define $\varphi: \bar{k}\left(\left(\Gamma_{c}\right)\right) \rightarrow \Gamma_{c} \cup\{\infty\}$ to be the valuation sending every $f$ in $\bar{k}\left(\left(\Gamma_{c}\right)\right)^{*}$ to $\min \operatorname{supp}(f)$ and $P_{\Gamma} \subset k((\Gamma))$ to be an ordering described in Proposition 2.1(iii). So in particular, $\left.\varphi\right|_{k((\Gamma))}$ is compatible with $P_{\Gamma}$. For convenience, let us fix the following notation: $K:=k\langle\Gamma\rangle ; K^{\prime}:=\bar{k}\left\langle\Gamma_{c}\right\rangle$; and $K^{\prime \prime}:=\bar{k}\left(\left(\Gamma_{c}\right)\right)$. As we have stated earlier, our objective is to construct suitable $\left(K, P^{\prime}\right), \varphi$, and $\sigma$. In view of the above results, we see that the ordered fields $\left(k((\Gamma)), P_{\Gamma}\right)$, $\left(k\langle\Gamma\rangle, P_{\Gamma} \cap k\langle\Gamma\rangle\right),\left(k(\Gamma), P_{\Gamma} \cap k(\Gamma)\right)$ with their respective compatible valuations $\left.\varphi\right|_{k((\Gamma))},\left.\varphi\right|_{k\langle\Gamma\rangle}$, and $\left.\varphi\right|_{k(\Gamma)}$ all satisfy (I) and (II). It turns out that $k\langle\Gamma\rangle$ is the one we want, because $k(\Gamma)$ is too "small" to admit some interesting order automorphism, and $k((\Gamma))$ is too "large" for defining an ordering we want.

In order to simplify the calculations needed later, we shall define an automorphism $\sigma^{\prime}$ on $\bar{k}\left\langle\Gamma_{c}\right\rangle$ such that its restriction on $k\langle\Gamma\rangle$ is the order automorphism $\sigma$ we want.

Proposition 2.4. $K^{\prime}$ is algebraically closed.

Proof. Let $L$ be a finite extension of $K^{\prime}$. As the residue field $\bar{K}_{\varphi \mid K^{\prime}}^{\prime} \cong \bar{k}$ is algebraically closed and the value group of $\left.\varphi\right|_{K^{\prime}}$ is divisible, it follows that $L$ is an immediate extension of $K^{\prime}$. Since $\operatorname{char} \bar{k}=0$ and $\left.\varphi\right|_{K^{\prime}}$ is Henselian by Proposition 2.2(i), by [Pr: Proposition 8.1 (ii)], we have $L=K^{\prime}$. Therefore $K^{\prime}$ is algebraically closed.

LEMMA 2.5. For any $s \in K^{*}$ with $\varphi(s)>0$ in $\Gamma, q \in \mathbb{N}$, the equation

$$
t^{q}=1+s
$$


has a unique solution in $1+I_{\varphi \mid K}$, where $I_{\varphi \mid K}=\{a \in K: \varphi(a)>0\}$. In fact it can be written in the form of $1+(s / q)+s^{\prime}$, for some $s^{\prime} \in K^{*}$ with $\varphi\left(s^{\prime}\right)>\varphi(s)$.

Proof. Again by Proposition 2.2, we see that $(K, \varphi \mid K)$ is Henselian. In the residue field $\bar{K}_{\varphi}, 1$ is a simple root of the equation $t^{q}=$ 1. Thus by the explicit calculation of Hensel's lemma, there exists a unique solution of the desired form.

From now on, we define $(1+s)^{1 / q}$ to be the solution obtained in the above lemma. Thus we can define $(1+s)^{p / q}:=\left((1+s)^{1 / q}\right)^{p}$, which of course also lies in $1+I_{\varphi \mid K}$. Obviously, $\left\{(1+s)^{p / q}: p / q \in \mathbb{Q}\right\}$ forms a multiplicative subgroup in $K^{*}$.

Lemma 2.6. For any $s \in K^{*}$ with $\varphi(s)=\alpha>0$, there exists a field automorphism $\sigma^{\prime}: K^{\prime} \rightarrow K^{\prime}$ such that

(i) $\sigma^{\prime}(x)=x(1+s)$,

(ii) $\left.\sigma^{\prime}\right|_{\bar{k}}=$ identity,

(iii) $\sigma:=\left.\sigma^{\prime}\right|_{K}$ is an automorphism of $K$,

(iv) $\varphi\left(\sigma^{\prime}(y)-y\right) \geq \varphi(y)+\alpha$ for all $y \in K^{* *}$.

Proof. Firstly, we shall construct a ring homomorphism $\lambda: \bar{k}\left[\Gamma_{c}\right] \rightarrow$ $\bar{k}\left\langle\Gamma_{c}\right\rangle$ such that (i), (ii) hold; $\lambda([\Gamma]) \subset K$ and (iv) holds for any element in $\bar{k}\left[\Gamma_{c}\right]$.

Since $\mathbb{Q} \subset \Gamma_{c}$, we can regard $\Gamma_{c}$ as a $\mathbb{Q}$ vector space. Let $I$ be a basis of $\Gamma_{c}$ over $\mathbb{Q}$. For convenience, we assume $1 \in I$. Also, we define $V:=\bigoplus_{\gamma \in I \backslash\{1\}} \mathbb{Q} \gamma$. So for any $a \in \Gamma_{c}$, there exists a unique $q_{a} \in \mathbb{Q}$ such that $a-q_{a} \in V$.

As $\bar{k}\left[\Gamma_{c}\right]$ and $K^{\prime}$ are $\bar{k}$ vector spaces, we can define a $\bar{k}$ vector space homomorphism $\lambda: \bar{k}\left[\Gamma_{c}\right] \rightarrow K^{\prime}$ such that

$$
\lambda\left(x^{a}\right)=(1+s)^{q_{a}} x^{a} .
$$

Clearly, $\lambda$ satisfies (i) and (ii). Next, it is straightforward to check that $\lambda \mid\left\{x^{a}: a \in \Gamma_{c}\right\}$ is a multiplicative group homomorphism. We therefore conclude that $\lambda$ is a ring homomorphism. As seen in the discussion following Lemma 2.5 , for any $p / q \in \mathbb{Q}^{*},(1+s)^{p / q}=$ $1+(p / q) s+s_{p / q} \in K$ for some $s_{p / q} \in K$ with $\varphi\left(s_{p / q}\right)>\varphi(s)=\alpha$. So for all $a \in \Gamma_{c}, \varphi\left(\lambda\left(x^{a}\right)-x^{a}\right) \geq a+\alpha$. Therefore (iv) holds for any element in $\bar{k}\left[\Gamma_{c}\right]$. Now, it follows from Proposition 2.3 that $\lambda$ extends to an automorphism $\sigma^{\prime}$ satisfying (iv). 
Lastly, observe also that $\lambda\left(x^{a}\right) \in K$ if $a \in \Gamma$. Therefore, by Proposition 2.3 again, the restriction of $\lambda$ on $k[\Gamma]$ extends to an automorphism on $K$ which must be $\left.\sigma^{\prime}\right|_{K}$.

REMARK. Note that if $\Gamma \subset \mathbb{Q}$, then $\Gamma_{c}=\mathbb{Q}$ and $\lambda\left(x^{a}\right)=(1+s)^{a} x^{a}$ for any $a \in \Gamma_{c}$.

3. The skew polynomial ring $K^{\prime}\left[t, \sigma^{\prime}\right]$. Let $\sigma^{\prime}, K, K^{\prime}, K^{\prime \prime}$, and $\varphi$ be as defined in $\S 2$ and $R^{\prime}:=K^{\prime}\left[t, \sigma^{\prime}\right]$. In $R^{\prime}$ multiplication is now induced by the rule $t \cdot a=\sigma^{\prime}(a) t$. For any $f(t), g(t) \in R^{\prime}$, let us denote the left polynomial $f(t) g(t)$ by $f * g(t)$. Since multiplication in $R^{\prime}$ is associative, $f *(g * h)(t)=(f * g) * h(t)$ for all $f(t), g(t), h(t) \in R^{\prime}$. In this section, we shall prove some lemmas needed later. In $k((\Gamma))$, we fix an element

$$
\omega=1+\sum_{i=1}^{\infty} \alpha_{i} x^{r_{i}},
$$

such that for all $i \in \mathbb{N}, \alpha_{i} \neq 0 ; 0<r_{1}<r_{2}<\cdots$ in $\Gamma$ and $\lim _{i \rightarrow \infty} r_{i}=r \leq \alpha / 2$. Note that such $\omega$ exists as $\Gamma$ is dense in $\mathbb{Q}$. Furthermore, it is easy to see that $\varphi(\omega)=0$ and $\omega \notin K^{\prime}$. Also, for any $f(t)=\sum_{i=0}^{n} a_{i} t^{i} \in R^{\prime}$, we define $f(\omega)=\sum_{i=0}^{n} a_{i} \omega^{i}$. Observe that as $K^{\prime}$ is algebraically closed and $\omega \notin K^{\prime}, f(\omega) \neq 0$ for any $f(t) \in R^{\prime} \backslash\{0\}$.

LEMMA 3.1. For any $y \in K^{\prime}$, we have $\varphi(\omega-y)<r$. In particular, $\varphi(\omega-y)<\varphi(y)+r$.

Proof. For any $y \in K^{\prime}, y$ can be written in the form of $\sum_{i=1}^{\infty} c_{i} x^{j_{t}}$ such that $\lim _{i \rightarrow \infty} j_{i}=\infty$. Hence $\left|\left\{j_{i}: j_{i}<r\right\}\right|$ is finite. Thus, there exists $g$ in $\operatorname{supp}(\omega-y)$ such that $g<r$. This clearly implies $\varphi(\omega-y)<r$. The second statement is a consequence of the first statement in case $\varphi(y)=0$. If $\varphi(y) \neq 0$, then

$$
\varphi(\omega-y)=\min (\varphi(y), 0) \leq \varphi(y)<\varphi(y)+r .
$$

LEMMA 3.2. For any $f(\omega)=\sum_{j=0}^{n} a_{j} \omega^{j} \in K^{\prime}[\omega]$, we define $f^{\sigma^{\prime}}(\omega)$ $=\sum_{j=0}^{n} \sigma^{\prime}\left(a_{j}\right) \omega^{j}$. Then $\varphi\left(f^{\sigma^{\prime}}(\omega)-f(\omega)\right)>\varphi(f(\omega))+\alpha-r$.

Proof. We prove this by induction on $\operatorname{deg} f$.

Suppose $\operatorname{deg} f=0$. Then the lemma follows from the construction of $\sigma^{\prime}$, since for all $u \in K^{\prime}, \varphi\left(\sigma^{\prime}(u)-u\right) \geq \varphi(u)+\alpha$. 
Let $f(\omega) \in K^{\prime}[\omega]$ be of degree $n$. As $K^{\prime}$ is algebraically closed, $f(\omega)=(\omega-y) g(\omega)$ for some $y \in K^{\prime}, g(\omega) \in K^{\prime}[\omega]$ with $\operatorname{deg} g=$ $n-1$. Clearly $f^{\sigma^{\prime}}(\omega)=\left(\omega-\sigma^{\prime}(y)\right) g^{\sigma^{\prime}}(\omega)$ and

$$
f^{\sigma^{\prime}}(\omega)-f(\omega)=\left(y-\sigma^{\prime}(y)\right) g^{\sigma^{\prime}}(\omega)+(\omega-y)\left[g^{\sigma^{\prime}}(\omega)-g(\omega)\right] .
$$

Therefore

$$
\begin{aligned}
& \varphi\left(f^{\sigma^{\prime}}(\omega)-f(\omega)\right) \\
& \quad \geq \min \left\{\varphi\left(y-\sigma^{\prime}(y)\right)+\varphi\left(g^{\sigma^{\prime}}(\omega)\right), \varphi(\omega-y)+\varphi\left(g^{\sigma^{\prime}}(\omega)-g(\omega)\right)\right\} .
\end{aligned}
$$

By construction of $\sigma^{\prime}$ and Lemma 3.1, we have

$$
\varphi\left(y-\sigma^{\prime}(y)\right) \geq \varphi(y)+\alpha>\varphi(\omega-y)+\alpha-r .
$$

By induction, we have

$$
\varphi\left(g^{\sigma^{\prime}}(\omega)-g(\omega)\right) \geq \varphi(g(\omega))+\alpha-r .
$$

In particular, Lemma 2.1 (iv) implies that $\varphi\left(g^{\sigma^{\prime}}(\omega)\right)=\varphi(g(\omega))$. Thus,

$$
\begin{aligned}
\varphi\left(f^{\sigma^{\prime}}(\omega)-f(\omega)\right) & \geq \varphi(\omega-y)+\varphi(g(\omega))+\alpha-r \\
& =\varphi(f(\omega))+\alpha-r .
\end{aligned}
$$

So the lemma is proved.

LEMMA 3.3. For any $f(t), g(t) \in R^{\prime} \backslash\{0\}$, we have

$$
\varphi(f * g(\omega)-f(\omega) g(\omega))>\varphi(f(\omega) g(\omega)) .
$$

In particular, $\varphi(f * g(\omega))=\varphi(f(\omega) g(\omega))$.

Proof. Without loss of generality, we may assume $f$ is monic. Again, we shall proceed by induction on $\operatorname{deg} f$.

If $f$ is a constant, then the lemma is trivial as now $f * g(\omega)=$ $f(\omega) g(\omega)$. Suppose $f(t)=t+c$ for some $c \in K^{\prime}$. Obviously,

$$
\begin{gathered}
f * g(\omega)=g^{\sigma^{\prime}}(\omega) \omega+c g(\omega) \text { and } \\
f * g(\omega)-f(\omega) g(\omega)=\left(g^{\sigma^{\prime}}(\omega)-g(\omega)\right) \omega .
\end{gathered}
$$

So

$$
\begin{gathered}
\varphi(f * g(\omega)-f(\omega) g(\omega))=\varphi\left(g^{\sigma^{\prime}}(\omega)-g(\omega)\right) \\
\geq \varphi(g(\omega))+\alpha-r \text { by Lemma } 3.2 .
\end{gathered}
$$

Note that $\alpha \geq 2 r$ and $r>\varphi(\omega+c)$ by Lemma 3.1. It follows that

$$
\varphi(f * g(\omega)-f(\omega) g(\omega)) \geq r+\varphi(g(\omega))+\alpha-2 r>\varphi(f(\omega) g(\omega)) .
$$


We now assume the lemma is proved for any $h(t)$ (not necessarily monic) in $R^{\prime}$ with its degree less than $n$.

Claim. For any $h(t), h^{\prime}(t) \in R^{\prime} \backslash\{0\}$, if both $\operatorname{deg} h, \operatorname{deg} h^{\prime}$ are less than $n$, then we have

$$
\varphi\left(h * h^{\prime} * g(\omega)-h(\omega) h^{\prime}(\omega) g(\omega)\right)>\varphi\left(h(\omega) h^{\prime}(\omega) g(\omega)\right) .
$$

Proof of Claim. By induction assumption, we have

$$
\begin{gathered}
\varphi\left(h *\left(h^{\prime} * g\right)(\omega)-h(\omega)\left(h^{\prime} * g\right)(\omega)\right)>\varphi(h(\omega))+\varphi\left(h^{\prime} * g(\omega)\right) \\
=\varphi(h(\omega))+\varphi\left(h^{\prime}(\omega)\right)+\varphi(g(\omega)),
\end{gathered}
$$

and

$$
\begin{aligned}
& \varphi\left(h(\omega) \cdot\left(h^{\prime} * g\right)(\omega)-h(\omega) \cdot h^{\prime}(\omega) g(\omega)\right) \\
& \quad=\varphi(h(\omega))+\varphi\left(\left(h^{\prime} * g\right)(\omega)-h^{\prime}(\omega) g(\omega)\right) \\
& >(h(\omega))+\varphi\left(h^{\prime}(\omega)\right)+\varphi(g(\omega)) .
\end{aligned}
$$

Combining the above inequalities, the desired inequality follows.

Let $f(t) \in R^{\prime}$ be of degree $n$. As before, $f(\omega)=(\omega-c) h(\omega)$ for some $c \in K^{\prime}$ and $h(t) \in R^{\prime}$ with $\operatorname{deg} h=n-1$. Let $f_{1}(t):=t-c$. By induction,

$$
\varphi\left(f(\omega)-f_{1} * h(\omega)\right)=\varphi\left((\omega-c) h(\omega)-f_{1} * h(\omega)\right)>\varphi(f(\omega)) .
$$

As both $f(t)$ and $f_{1} * h(t)$ are monic, $\operatorname{deg}\left(f-f_{1} * g\right) \leq n-1$. Therefore by induction again,

$$
\varphi\left(\left(f-f_{1} * h\right) * g(\omega)\right)=\varphi\left(f(\omega)-f_{1} * h(\omega)\right)+\varphi(g(\omega)) .
$$

Hence

$$
\begin{aligned}
\varphi\left(f * g(\omega)-f_{1} * h * g(\omega)\right)=\varphi\left(\left(f-f_{1} * h\right) * g(\omega)\right) \\
\quad=\varphi\left(f(\omega)-f_{1} * h(\omega)\right)+\varphi(g(\omega)) \\
>\varphi(f(\omega))+\varphi(g(\omega)) .
\end{aligned}
$$

On the other hand, by the above claim, we have

$$
\varphi\left(f_{1} * h * g(\omega)-(\omega-c) h(\omega) g(\omega)\right)>\varphi(f(\omega))+\varphi(g(\omega)) .
$$

Hence by combining the above two inequalities, we get

$$
\varphi(f * g(\omega)-f(\omega) g(\omega))>\varphi(f(\omega))+\varphi(g(\omega)) .
$$

4. The final step. Let $R$ be the subring generated by $K$ and $t$ in $R^{\prime}$. Since $\sigma=\left.\sigma^{\prime}\right|_{K}$ is an automorphism, $R \cong K\left[t^{\prime}, \sigma\right]$. As $\sigma$ is an 
automorphism, it is well known that $R$ is both right Ore and left Ore [C: Proposition 1.3.1 and Proposition 1.3.2]. Let $D:=\left\{x y^{-1}: x, y \in\right.$ $R$ and $y \neq 0\}$ be the right quotient ring of $R$. As is well known, $D$ is a division ring. Firstly, we shall define an ordering on $D$.

Proposition 4.1. $P:=\left\{f(t) g(t)^{-1}: g(t), f(t) \in R, g(t) \neq 0\right.$ and $\left.f(\omega) g(\omega) \in P_{\Gamma}\right\}$ is an ordering on $D$.

Proof. To show $P$ is an ordering, it suffices to show that $P^{\prime}:=$ $\left\{f(t) \in R: f(\omega) \in P_{\Gamma}\right\}$ is an ordering on $R$ by [F: Chapter 6, Theorem 3]. Obviously, $P^{\prime} \cup\left(-P^{\prime}\right)=R$ and $P^{\prime}$ is closed under addition. As we have seen earlier, for any $f(t) \in R, 0=f(t) \in R$ if and only if $f(\omega)=0$ in $k((\Gamma))$. This certainly implies $P^{\prime} \cap\left(-P^{\prime}\right)=\{0\}$. This it remains to show that $P^{\prime}$ is closed under multiplication.

Suppose $f(t), g(t) \in P^{\prime} \backslash\{0\}$. Thus, $f(\omega), g(\omega) \in P_{\Gamma} \backslash\{0\}$. On the other hand, by Lemma 3.3 we have $\varphi(f * g(\omega)-f(\omega) g(\omega))>$ $\varphi(f(\omega) g(\omega))$. It follows from Proposition 2.1 (iv) that $f * g(\omega)>0$ in $k((\Gamma))$. Hence $P^{\prime}$ is closed under multiplication.

REMARK. From now on, we shall write $a>0$ if $a \in P \backslash\{0\}$.

LEMMA 4.2. Let $v^{\prime}: R \rightarrow \Gamma \cup\{\infty\}$ be a mapping such that for any $f(t) \in R, v^{\prime}(f(t))=\varphi(f(\omega))$. Then for any $f(t), g(t) \in R$, we have

(i) $v^{\prime}(f(t))=\infty$ if and only if $f(t)=0$.

(ii) $v^{\prime}(f(t) g(t))=v^{\prime}(f(t))+v^{\prime}(g(t))$.

(iii) $v^{\prime}(f(t)+g(t)) \geq \min \left\{v^{\prime}(f(t)), v^{\prime}(g(t))\right\}$.

(iv) $0<f(t)<g(t)$ in $(D, P)$, implies $v^{\prime}(g(T)) \leq v^{\prime}(f(t))$.

Proof. (i) follows from the fact $\omega$ is transcendental over $K^{\prime}$ and (iii) from the fact that $\varphi$ is a valuation on $\bar{k}\left(\left(\Gamma_{c}\right)\right)$. For (ii) and (iv), we take any $f(t), g(t) \in R$. Obviously,

$$
\begin{aligned}
v^{\prime}(f(t) g(t)) & =v^{\prime}(f * g(t))=\varphi(f * g(\omega)) \\
& =\varphi(f(\omega) g(\omega))=v^{\prime}(f(t))+v^{\prime}(g(t)) .
\end{aligned}
$$

If $0<f(t)<g(t)$ in $(D, P)$, then $0<f(\omega)<g(\omega)$ in $\left(k((\Gamma)), P_{\Gamma}\right)$. Thus by compatibility of $\left.\varphi\right|_{k((\Gamma))}$ and $P_{\Gamma}, \varphi(f(\omega)) \geq \varphi(g(\omega))$. Clearly this implies $v^{\prime}(f(t)) \geq v^{\prime}(g(t))$.

Theorem 4.3. Let $D, P, v^{\prime}$ be as above. Then $v^{\prime}$ extends to a valuation $v: D \rightarrow \Gamma \cup\{\infty\}$ compatible with $P$. Moreover, the ordered residue division ring $\left(\bar{D}_{v}, \bar{P}_{v}\right)$ is order isomorphic to $\left(k, P_{k}\right)$. 
Proof. Let us define $v: D \rightarrow \Gamma \cup\{\infty\}$ such that

$$
v(0)=\infty \quad \text { and } \quad v\left(x y^{-1}\right)=v^{\prime}(x)-v^{\prime}(y) \quad \forall x, y \in R \backslash\{0\} .
$$

Firstly, we show that $v$ is well defined. Let $x, y \in R \backslash\{0\}$. As $R$ is also a left Ore domain, there exist $a, b \in R \backslash\{0\}$ such that $a x=b y$. If $x y^{-1}=x^{\prime} y^{\prime-1}$ for some $x^{\prime}, y^{\prime} \in R \backslash\{0\}$, then we also have $a x^{\prime}=$ $b y^{\prime}$. By Lemma 4.1 (i), $v^{\prime}(x)-v^{\prime}(y)=v^{\prime}(a)-v^{\prime}(b)=v^{\prime}\left(x^{\prime}\right)-v^{\prime}\left(y^{\prime}\right)$. Thus $v$ is well defined.

Let $x, y, x^{\prime}, y^{\prime} \in R \backslash\{0\}$. Since $R$ is also right Ore, there exist $a^{\prime}, b^{\prime} \in R \backslash\{0\}$ such that $y a^{\prime}=x^{\prime} b^{\prime}$. Obviously, $v^{\prime}\left(x^{\prime}\right)-v^{\prime}(y)=$ $v^{\prime}\left(a^{\prime}\right)-v^{\prime}\left(b^{\prime}\right)$ by Lemma 4.2(ii). On the other hand, it is easy to see that $x y^{-1} \cdot x^{\prime} y^{\prime-1}=x a^{\prime} b^{\prime-1} y^{\prime-1}=\left(x a^{\prime}\right)\left(y^{\prime} b^{\prime}\right)^{-1}$ in $D$. Thus

$$
\begin{aligned}
v\left(x y^{-1} \cdot x^{\prime} y^{\prime-1}\right) & =v\left(\left(x a^{\prime}\right)\left(y^{\prime} b^{\prime}\right)^{-1}\right)=v^{\prime}\left(x a^{\prime}\right)-v^{\prime}\left(y^{\prime} b^{\prime}\right) \\
& =v^{\prime}(x)+v^{\prime}\left(a^{\prime}\right)-v^{\prime}\left(b^{\prime}\right)-v^{\prime}\left(y^{\prime}\right) \\
& =v\left(x y^{-1}\right)+v\left(x^{\prime} y^{\prime-1}\right) .
\end{aligned}
$$

Next, we let $c, d, w \in R \backslash\{0\}$ be such that $y c=w, y^{\prime} d=w \in$ $R$. Hence $x y^{-1} w=c x, x^{\prime} y^{\prime-1} w=x^{\prime} d$ and $x y^{-1}+x^{\prime} y^{\prime-1}=$ $\left(x c+x^{\prime} d\right) w^{-1}$. Thus

$$
\begin{aligned}
v\left(x y^{-1}+x^{\prime} y^{\prime-1}\right)=v\left(\left(x c+x^{\prime} d\right) w^{-1}\right) \\
\quad=v^{\prime}\left(x c+x^{\prime} d\right)-v^{\prime}(w) \\
\quad \geq \min \left\{v^{\prime}(x c), v^{\prime}\left(x^{\prime} d\right)\right\}-v(w) \\
\quad=\min \left\{v^{\prime}(x)+v^{\prime}(c)-v^{\prime}(w), v^{\prime}\left(x^{\prime}\right)+v^{\prime}(d)-v^{\prime}(w)\right\} \\
\quad=\min \left\{v^{\prime}(x)-v^{\prime}(y), v^{\prime}\left(x^{\prime}\right)-v^{\prime}\left(y^{\prime}\right)\right\} \\
\quad=\min \left\{v\left(x y^{-1}\right), v\left(x^{\prime} y^{\prime-1}\right)\right\} .
\end{aligned}
$$

Concluding from above, we see that $v$ is a valuation on $D$. Note that the surjectivity of $v$ follows easily from the fact that $\left.v\right|_{K}=\left.\varphi\right|_{K}$. Let us determine the residue division ring. Suppose $f(t), g(t) \in R \backslash\{0\}$ and $v\left(f(t) g(t)^{-1}\right)=0$. By definition of $v$, we have $\varphi\left(f(\omega) g(\omega)^{-1}\right)$ $=0$. Since both $f(\omega), g(\omega) \in K((\Gamma))$ and $k$ is projected onto the residue field of $\left(k((\Gamma)),\left.\varphi\right|_{k((\Gamma))}\right)$, there exists $a \in k$ such that $\varphi\left(f(\omega) g(\omega)^{-1}-a\right)>0$. Therefore $v\left(f(t) g(t)^{-1}-a\right)>0$. It follows that the projection of $k$ on the residue field of $(D, v)$ is also surjective. Lastly, it remains to show that $v$ is compatible with $P$. It is enough to show that if $v\left(x y^{-1}\right)>0$, then $1+x y^{-1} \in P$. Without loss of generality, we may assume $y>0$. As $v\left(x y^{-1}\right)=v^{\prime}(x)-v^{\prime}(y)>0$, we have $v^{\prime}(x)>v^{\prime}(y)$. By Lemma 4.2(iv), it follows that $y>|x|$. Hence $y+x>0$ and $1+x y^{-1}=(y+x) y^{-1}>0$ in $(D, P)$. 
REMARK. In our construction, we have a copy of $k$ lying inside the center, whereas in Schröder's construction, there may not exist a copy of $k$ lying inside the center.

Acknowledgment. This work represents a portion of the author's Ph.D dissertation done at the University of California, Berkeley. The author would like to thank his advisor Professor T. Y. Lam for his guidance and encouragement.

\section{REFERENCES}

[C] P. M. Cohn, Skew Field Constructions, London Math. Soc., Lecture Note Series 27, 1973.

[E] O. Endler, Valuation Theory, Springer Verlag, Berlin and New York, 1972.

[F] L. Fuchs, Partially Ordered Algebraic Systems, Pergamon Press, Oxford, London, New York, Paris, 1963.

[N] B. H. Neumann, On ordered division rings, Trans. Amer. Math. Soc., 66 (1949), 202-252.

[Pr] A. Prestel, Lectures on Formally Real Fields, Lecture Notes in Mathematics, 1093, Springer-Verlag, 1984.

[P] S. Priess-Crampe, Angeordnete Strukturen: Gruppen, Körper, Projektive Ebenen, Ergebnisse der Mathematik und ihrer Grenzgebiete 98 Springer-Verlag, Berlin, Heidelberg, New York, Tokyo, 1983.

[S] O. Schilling, The Theory of Valuations, Amer. Math. Soc., Mathematical Survey 4 (1950).

[M] M. Schröder, Angeordnete Schiefkörper mit Natürlicher Bewertung vom Rang 1, Schriftenreihe der Mathematischen Institut der Universität Münster, 2 Serie, Heft 39, 1986.

[T1] A. Tschimmel, Über Anordnungsräume von Schiefkörper, Dissertation, Unversistät Münster, 1981.

[T2] __ Lokal-Global Prinzipien für Anordnungen bewerteter Schiefkörper, Arch. Math., 44 (1985), 48-58.

Received January 26, 1989 and in revised form October 22, 1989.

National UNiversity of Singapore

10 Kent Ridge CRESCENT

SINGAPORE 0511 



\section{PACIFIC JOURNAL OF MATHEMATICS EDITORS}

V. S. VARADARAJAN

(Managing Editor)

University of California

Los Angeles, CA 90024-1555-05

Herbert Clemens

University of Utah

Salt Lake City, UT 84112

THOMAS ENRIGHT

University of California, San Diego

La Jolla, CA 92093

\section{R. FINN}

Stanford University

Stanford, CA 94305

HermanN FlaschKa

University of Arizona

Tucson, AZ 85721

VAUGHAN F. R. JoNES

University of California

Berkeley, CA 94720

STEVEn KeRCKHOFF

Stanford University

Stanford, CA 94305
C. C. MOORE

University of California

Berkeley, CA 94720

MARTIN SCHARLEMANN

University of California

Santa Barbara, CA 93106

Harold STARK

University of California, San Diego La Jolla, CA 92093

\section{ASSOCIATE EDITORS}

R. ARENS

E. F. BECKENBACH (1906-1982)
B. H. NeumanN

\section{SUPI}

UNIVERSITY OF ARIZONA

UNIVERSITY OF BRITISH COLUMBIA

CALIFORNIA INSTITUTE OF TECHNOLOGY

UNIVERSITY OF CALIFORNIA

MONTANA STATE UNIVERSITY

UNIVERSITY OF NEVADA, RENO

NEW MEXICO STATE UNIVERSITY

OREGON STATE UNIVERSITY
F. WolF
K. YoshidA
(1904-1989)

UNIVERSITY OF OREGON UNIVERSITY OF SOUTHERN CALIFORNIA

STANFORD UNIVERSITY

UNIVERSITY OF HAWAII

UNIVERSITY OF TOKYO

UNIVERSITY OF UTAH

WASHINGTON STATE UNIVERSITY

UNIVERSITY OF WASHINGTON 


\section{Pacific Journal of Mathematics}

Vol. 147, No. $1 \quad$ January, 1991

Mark S. Ashbaugh, Evans Malott Harrell, II and Roman Svirsky, On minimal and maximal eigenvalue gaps and their causes $\ldots \ldots \ldots \ldots \ldots 1$

Robert Coleman and Francis Oisin McGuinness, Rational formal group

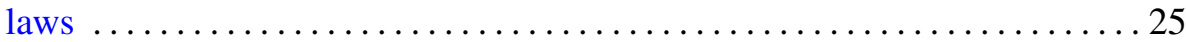

Jacek M. Cygan and Leonard Frederick Richardson, $D$-harmonic distributions and global hypoellipticity on nilmanifolds ...........29

Satya Deo and Kalathoor Varadarajan, Some examples of nontaut

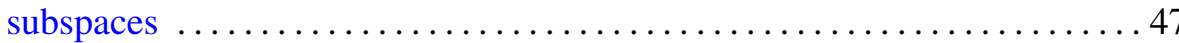

Maria Fragoulopoulou, Automatic continuity of *-morphisms between

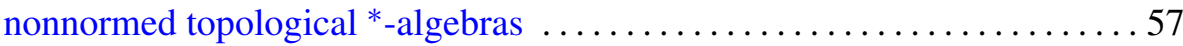

Stephen J. Gardiner, Removable singularities for subharmonic functions . . . 71 Herbert Paul Halpern, Victor Kaftal and László Zsidó, Finite weight projections in von Neumann algebras $\ldots \ldots \ldots \ldots \ldots \ldots \ldots \ldots \ldots \ldots$

Telemachos E. Hatziafratis, Explicit $\bar{\partial}$-primitives of Henkin-Leiterer kernels on Stein manifolds

Ka Hin Leung, A construction of an ordered division ring with a rank one

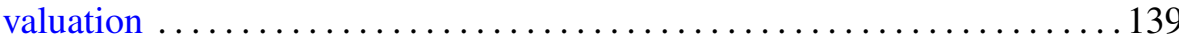

Christopher K. McCord, Nielsen numbers and Lefschetz numbers on solvmanifolds

Katsuro Sakai and Raymond Y. T. Wong, Manifold subgroups of the homeomorphism group of a compact $Q$-manifold

Caroline Perkins Sweezy, $L$-harmonic functions and the exponential square class 